\title{
Chronic gastritis (Clinical case)
}

\author{
Gastrite crónica (Caso clínico)
}

Bruno Sousa $^{(1,2,3)}$, Nelson Tavares ${ }^{(1,2)}$

${ }^{1}$ School of Sciences and Health Technologies, Universidade Lusófona de Humanidades e Tecnologias, Lisboa, Portugal

${ }^{2} \mathrm{CBIOS}$ - Research Center for Biosciences and Health Technologies

${ }^{3}$ Health Service of Autonomous Region of Madeira

e-mail: bruno.sousa@mail.com

A 42-year-old heavy-duty driver (from 9 am to $5 \mathrm{pm}$ ) with a part-time as a cook (7 pm to 11:30 pm), who has complaints of dyspepsia. He was sent to the Nutrition Consultation by his General Practitioner with Sucralfate, Diazepan and Esomeprazole and diagnosed with chronic gastritis.

\section{Anthropometric evaluation}

Weight: $66.4 \mathrm{~kg}$

Height: $173 \mathrm{~cm}$

BMI: $22.2 \mathrm{~kg} / \mathrm{m}^{2}$

Waist circumference: $84 \mathrm{~cm}$

Assessment of body composition (TANITA TBF 300 ${ }^{\circledR}$ )

Body fat: $17.2 \%$

Fatty mass: $11.4 \mathrm{~kg}$

Fat free mass: $55 \mathrm{~kg}$

Total body water: $40.3 \mathrm{~kg}$

\section{Analytical Parameters:}

\section{Hematology}

Leukocytes: $7.8 \times 10^{3} / \mu \mathrm{L}(4.5-110)$

Erythrocytes: $4.37 \times 10^{6} / \mu \mathrm{L}(4.50-6.50)$

Hemoglobin: 13.1g / dL (13.0 - 18.0)

Hematocrit: $39.0 \%$ (40.0 - 54.0)

Glycated hemoglobin (A1C) - 5.8\% (4.0 - 6.0)

\section{Biochemistry}

Glucose: $102.0 \mathrm{mg} / \mathrm{dL}(74.0$ - 110.0)

Urea: $44.5 \mathrm{mg} / \mathrm{dL}(8.0-50.0)$

Creatinine: $0.93 \mathrm{mg} / \mathrm{dL}(0.70-1.20)$

Uric acid $5.4 \mathrm{mg} / \mathrm{dL}(4.8$ - 8.7)

Total cholesterol: $225 \mathrm{mg} / \mathrm{dL}(<200.0)$

HDL cholesterol: $39.0 \mathrm{mg} / \mathrm{dL}$ (40.0 - 60.0)

LDL cholesterol: $162.4 \mathrm{mg} / \mathrm{dL}(<175.0)$
Received /Recebido: 29/10/2018 Accepted / Aceite: 19/11/2018

Electronic Edition: www.alies.pt

Homem de 42 anos, motorista de veículos pesados (das $9 \mathrm{~h}$ às $17 \mathrm{~h}$ ) com um part-time como cozinheiro (das $19 \mathrm{~h}$ às 23h30), e que apresenta queixas de dispepsia. Foi enviado à Consulta de Nutrição pela médica de família por gastrite crónica, medicado com Sucralfato, Diazepam e Esomeprazol.

Avaliação antropométrica

Peso: $66,4 \mathrm{~kg}$

Estatura: $173 \mathrm{~cm}$

IMC: $22,2 \mathrm{~kg} / \mathrm{m}^{2}$

Perímetro da cintura: $84 \mathrm{~cm}$

Avaliação da composição corporal (TANITA TBF $300 \circledR)$

Gordura corporal: $17,2 \%$

Massa gorda: $11,4 \mathrm{~kg}$

Massa isenta de gordura: $55 \mathrm{~kg}$

Água corporal total: $40,3 \mathrm{~kg}$

\section{Parâmetros analíticos:}

Hematologia

Leucócitos: $7,810^{\wedge} 3 / \mu \mathrm{L}(4,5-110)$

Eritrócitos: $4,3710^{\wedge} 6 / \mu \mathrm{L}(4,50-6,50)$

Hemoglobina: $13,1 \mathrm{~g} / \mathrm{dL}(13,0-18,0)$

Hematócrito: $39,0 \%(40,0-54,0)$

Hemoglobina glicada (A1C) - 5,8\% $(4,0-6,0)$

Bioquímica

Glicose: $\mathbf{1 0 2 , 0 m g / d L ~ ( 7 4 , 0 ~ - ~ 1 1 0 , 0 ) ~}$

Ureia: $44.5 \mathrm{mg} / \mathrm{dL}(8,0-50,0)$

Creatinina: 0,93mg/dL $(0,70-1,20)$

Ácido úrico $5,4 \mathrm{mg} / \mathrm{dL}(4,8-8,7)$

Colesterol total: $225 \mathrm{mg} / \mathrm{dL}(<200,0)$

Colesterol HDL: $39,0 \mathrm{mg} / \mathrm{dL}(40,0-60,0)$ 
Triglycerides: $118.0 \mathrm{mg} / \mathrm{dL}(<150.0)$

Alanine Aminotransferase: 11.4U / L (17.0-63.0)

Aspartate Aminotransferase: 13.5U / L (10.0-50.0)

Gamaglutamyltransferase: 17.4U / L (7.0-50.0)

Pancreatic amylase: $26.3 \mathrm{U} / \mathrm{L}(<46.0)$

Iron: $97.0 \mu \mathrm{g} / \mathrm{dL}$ (45.0 - 182.0)

Hormonology

Thyroid Function:

Free T4: $1.2 \mathrm{ng} / \mathrm{dL}(0.6$ - 1.7)

TSH: $1.54 \mu \mathrm{UI} / \mathrm{mL}(0.30$ - 4.70)

Anemias:

Ferritin: $256.1 \mathrm{ng} / \mathrm{mL}(30.0$ - 400.0)

Folic acid: $4.65 \mathrm{ng} / \mathrm{mL}(>4.60)$

Vitamin B12: 275 pg / mL (197-771)

Clinical evaluation:

Personal background:

Mixed dyslipidemia

Had a stroke for about 10 years

Medication:

Esomeprazol, Diazepam, Sucralfate

Recent screening tests:

Upper gastrointestinal endoscopy: chronic gastritis, moderate active

Intestinal transit: regular

Eating habits:

Wake up at 6:30 a.m.

Breakfast:

Does not take

During the morning:

3 espresso coffees with 1 packet of sugar (each)

Lunch: 1:30 p.m.

Dish: Half a plate of white rice or 2 potatoes + meat or

fish $(120 \mathrm{~g})+$ cooked vegetables $(50 \mathrm{~g})$

Drink soda $(200 \mathrm{ml})$

1 espresso coffee with 1 packet of sugar

During the afternoon:

3 espresso coffees with 1 packet of sugar (each)

Dinner: 7:30 p.m.

Identical to lunch

1 espresso coffee with 1 packet of sugar

Between dinner and bedtime:

2 espresso coffees with 1 packet of sugar (each)

Goes to bed at midnight

Water consumption: about $1 \mathrm{~L}$ per day

Consumption of soluble cereal drink with coffee: $750 \mathrm{ml}$ per day

He had accentuated ethanolic habits. Refers to abstinence for about 6 months.

$\mathrm{He}$ is a smoker (about 10 cigarettes per day) for many years.

Environment, behavior and social:

He takes many meals outside of his home and refers
Colesterol LDL: $162,4 \mathrm{md} / \mathrm{dL}(<175,0)$

Triglicéridos: 118,0mg/dL $(<150,0)$

Alanina Aminotransferase: 11,4U/L $(17,0-63,0)$

Aspartato Aminotransferase: $13,5 \mathrm{U} / \mathrm{L}(10,0-50,0)$

Gamaglutamiltransferase: 17,4U/L $(7,0-50,0)$

Amilase pancreática: 26,3 U/L $(<46,0)$

Ferro: $97,0 \mu \mathrm{g} / \mathrm{dL}(45,0-182,0)$

Hormonologia

Função tiroideia:

T4 livre: $1,2 \mathrm{ng} / \mathrm{dL}(0,6-1,7)$

TSH: $1,54 \mu \mathrm{UI} / \mathrm{mL}(0,30-4,70)$

Anemias:

Ferritina: $256,1 \mathrm{ng} / \mathrm{mL}(30,0-400,0)$

Ácido fólico: 4,65ng/mL $(>4,60)$

Vitamina B12: 275pg/mL (197 - 771)

Avaliação clínica:

Antecedentes pessoais:

Dislipidemia mista

Acidente vascular cerebral há cerca de 10 anos

Medicação:

Esomeprazol, Diazepam, Sucralfato.

Exames complementares de diagnóstico recentes:

Endoscopia digestiva alta: gastrite crónica, ativa moderada

Trânsito intestinal: regular

Hábitos alimentares:

Acorda às $6 \mathrm{~h} 30$

Pequeno-almoço:

Não faz

Durante a manhã:

3 cafés expresso com 1 pacote de açúcar (cada um)

Almoço: $13 \mathrm{~h} 30$

Prato: Meio prato de arroz branco ou 2 batatas + carne ou peixe $(120 \mathrm{~g})+$ verdura cozidas $(50 \mathrm{~g})$

Bebe refrigerante $(200 \mathrm{ml})$

1 café expresso com 1 pacote de açúcar

Durante a tarde:

3 cafés expresso com 1 pacote de açúcar (cada um)

Jantar: $19 \mathrm{~h} 30$

Idêntico ao almoço

1 café expresso com 1 pacote de açúcar

Entre o jantar e o deitar:

2 cafés expresso com 1 pacote de açúcar (cada)

Deita-se às $24 \mathrm{~h}$

Consumo de água: cerca de 1L por dia

Consumo de bebida solúvel de cereais com café: $750 \mathrm{ml}$ por dia

Apresentava hábitos etílicos acentuados. Refere abstinência há cerca de 6 meses.

É fumador (cerca de 10 cigarros por dia) há muitos anos.

Ambiente, comportamento e social:

Faz muitas refeições extra domicílio e refere que tem 
that he has a strong preference for fast food and for foods high in fat.

He refers that while he is cooking, he tends to snack.

He says that he does not eat while driving and does not like to eat in the morning and in the afternoon.

He does not forgo coffee consumption.

He has a sedentary activity and shows no desire to change this lifestyle.

Questions:

1. Is chronic gastritis a serious disease?

2. Is the absortion of essential vitamins compromised in chronic gastritis?

3. And what about other micronutrients?

4. What foods should be avoided?

5. Consumption of what foods should be increased?

Answers:

1. The significance of chronic gastritis as a serious disease is largely underrated in clinical practice, even though the role of gastritis in the pathogenesis of ordinary peptic ulcers and gastric cancers is obvious.

2. In an acid-free and atrophic stomach, the absorption of the essential vitamins, like vitamin B12, are severely compromised due to the impairment in secretion of intrinsic factor.

3. Dietary metabolism and absorption of micronutrients, like iron, calcium, magnesium and zinc are impaired in acid-free stomach too.

4. Alcohol, beer, wine and spirits. Spices, particularly red and black peppers. Coffee and caffeine.

5. Omega-3 and omega- 6 fatty acids which may have a protective effect. uma grande preferência por fast food e por alimentos ricos em gordura.

Enquanto está a cozinhar, tem por hábito petiscar.

Afirma que não come enquanto conduz nem gosta de comer durante a manhã e a tarde.

Não dispensa o consumo de café.

Apresenta uma atividade sedentária e não mostra vontade em alterar este estilo de vida.

Questões:

1. A Gastrite crónica é uma doença grave?

2. A absorção de vitaminas essenciais falha quando existe gastrite crónica?

3. E o que acontece com outros micronutrientes?

4. Que alimentos devem ser evitados?

5. Deve ser aumentado o consumo de que alimentos?

Respostas:

1. O significado da gastrite crónica como uma doença grave é amplamente subestimado na prática clínica, embora o papel da gastrite na patogénese das úlceras pépticas comuns e dos cancros gástricos seja óbvio.

2. Num estômago atrófico e sem ácido, devido ao comprometimento da secreção do fator intrínseco, a absorção das vitaminas essenciais, como a vitamina B12, falha severamente.

3. O metabolismo da dieta e a absorção de micronutrientes, como ferro, cálcio, magnésio e zinco, também são prejudicados no estômago sem ácido.

4. Álcool, cerveja, vinho e bebidas espirituosas. Especiarias, particularmente pimenta vermelha e preta. Café e cafeína.

5. Ácidos gordos ómega-3 e ómega-6, que podem ter um efeito protetor.

\section{References/Referências}

IARC Schistosomes, liver flukes and Helicobacter pylori. IARC Working Group on the Evaluation of Carcinogenic Risks to Humans. Lyon, 7-14 June 1994. IARC Monogr Eval Carcinog Risks Hum. 1994;61:1-241.

Schindler R. Chronic gastritis. Klin Wochenschr. 1966;44:601-12.

Siurala M. The story of gastritis. Scand J Gastroenterol Suppl. 1991;186:1-3.

Telaranta-Keerie A, Kara R, Paloheimo L, Härkönen M, Sipponen P. Prevalence of un

diagnosed advanced atrophic corpus gastritis in Finland: an observational study among 4,256 volunteers without specific complaints. Scand J Gastroenterol. 2010;45:1036-41.

Sipponen P, Laxén F, Huotari K, Härkönen M. Prevalence of low vitamin B12 and high homocysteine in serum in an elderly male population: association with atrophic gastritis and Helicobacter pylori infection. Scand J Gastroenterol. 2003;38:1209-16.

Mahan K, Escott-Stump S, Raymond J. Krause’s food \& nutrition care process. 13th ed. Philadelphia: Saunders, 2011. 